Results 32 children (18 boys, 14 girls) were identified who required PN for intestinal failure for combined total of over 12,500 PN days. 9 children had no positive blood cultures. There were 126 positive blood cultures (27 organisms isolated) in the remaining 23 children. Of the 21 children who used a heparin-saline based catheter lock, $86 \%$ had one or more CRBSI. 11 children used a taurolidine-based catheter lock, with only $45 \%$ having one or more CRBSI.

Conclusion There was a significant reduction in the incidence of CRBSIs in those children using taurolidine-based catheter locks $\left(\right.$ TauroLock $^{\mathrm{TM}}$ ) compared to heparin locks. There was an absolute risk reduction of $40.3 \%$ (95\% CI $7.25-73.3 \%$ ) with a numbers needed to treat (NNT) of 3 (95\% CI 1.4-13.8). The use of taurolidine locks on all children on long-term home PN could reduce morbidity and morality, and have a significant impact on the associated costs of CRBSIs. Taurolidine-based catheter locks should be considered for all children on long-term PN.

\section{G205(P) STOOL SHORT CHAIN FATTY ACID CONCENTRATIONS IN A COHORT OF PRETERM VERY LOW BIRTH WEIGHT INFANTS WITH AND WITHOUT NECROTISING ENTEROCOLITIS}

doi:10.1136/archdischild-2013-304107.217

'LM Beattie, ${ }^{2} \mathrm{~K}$ Gerasimidis, ${ }^{2} \mathrm{CA}$ Edwards, ${ }^{2} \mathrm{AR}$ Barclay, ${ }^{1} \mathrm{JH}$ Simpson, ${ }^{2} \mathrm{DJ}$ Morrison. ${ }^{1}$ Neonatal Unit, Royal Hospital for Sick Children, Yorkhill, UK; ${ }^{2}$ Department of Child Health, University of Glasgow, UK

Introduction Diagnostic markers of necrotising enterocolitis (NEC) remain evasive. Stool short chain fatty acids (sSCFAs) are a product of bacterial fermentation of undigested carbohydrate and protein noted to alter in animal models of NEC. According to the Lawrence Hypothesis, they may be causative of NEC. We sought to correlate changes in sSCFAs over the first month of life in a cohort of preterm, very low birth weight infants with and without NEC.

Methods 56 sequentially recruited infants $<32$ weeks and $<1.5 \mathrm{Kg}$ birth weight within week 1 of life. Stool samples taken once weekly for the first 4 weeks, analysed by gas chromatography-mass spectrometry ( $\mathrm{mcg} / \mathrm{g}$ wet weight). 11 individual acids were measured: acetate, lactate, isobutyrate, butyrate, isocaproate, caproate, isovalerate, valerate, octanoate, heptanoate and lactate. NEC was diagnosed by consultant, external collaborator and radiologist, using Bell's Criteria

Results $\mathrm{N}=56$ infants ( $83 \%$ recruitment). 20 developed $\geq$ Bell's $2 \mathrm{a}$. 8 required surgery (5 ileostomy). Further clinical/demographical information can be found in abstract BEAT82431. There were no correlations between gestation, feed, NEC and sSCFAs. No significant differences were observed in weekly totals. Wide interquartile ranges were noted (Week 1: $20.9 \pm 26$; Week 2: $15.8 \pm 19.1$; Week 3: $13.2 \pm 20.8$; Week 4: $12 \pm 22.9$ ). Acetate and lactate dominated each sample, regardless of gestation, feed or NEC $(p<0.05)$. Subgroup analysis revealed significant differences in stage $2 a$ and $3 b$ NEC. Stage 2a showed higher concentrations of propionate in week 4 than week $3(0.74 \pm 6.45 \mathrm{Vs} 0.15 \pm 0.17, \mathrm{p}=0.05 \mathrm{MWU})$, and lower valerate in week 4 than $2(0.00476 \pm 0.012$ Vs $0.0129 \pm 0.028$ $\mathrm{p}=0.02 \mathrm{MWU})$. Stage $3 \mathrm{~b}$ isobutyrate and heptanoate concentrations were significantly lower in week 4 than 3 (I: $0.007 \pm 0.026 \mathrm{Vs}$ $0.053 \pm 0.09, p=0.03 ; \mathrm{H}: 0.011 \pm 0.013 \mathrm{Vs} 0.023 \pm 0.043, \mathrm{p}=0.03)$.

Conclusion Despite a wide variation in clinical status, the levels of sSCFAs remained remarkably consistent. Small yet significant differences in minor sSCFAs were seen in subgroup analysis in those with stage $2 a$ and $3 b$ NEC. Reasons for the high incidence of NEC require further investigation.

\section{G206(P) THE IMPACT OF ESPGHAN GUIDELINES ON THE INVESTIGATIONS FOR COELIAC DISEASE}

doi:10.1136/archdischild-2013-304107.218
'M Bhardwaj, 'H Banoub, 'N Sumar, 'M Lawson, 'S Chong. 'Paediatric Gastroenterology, Queen Marys Hospital for Children, Carshalton, UK; ${ }^{2}$ Immunology, St Heliers Hospital, Carshalton, UK

Background Coeliac Disease (CD) is an immune-mediated systemic disorder elicited by gluten and related prolamines in genetically susceptible individuals. ${ }^{1}$ The diagnosis of $\mathrm{CD}$ depends on gluten dependant symptoms; CD-specific antibodies - against TG2, endomysial antibodies (EMA), and deamidated forms of gliadin peptides (DGP); the presence of HLA-DQ2/HLA-DQ8 and characteristic histological changes in duodenal biopsy. ESPGHAN guidelines suggest histological assessment may be omitted where clinical symptoms may be attributed to $\mathrm{CD}$ in addition to a high IgA anti-tTG levels ( $>10$ times the upper limits of normal for the reference laboratory), verified by EMA positivity and HLA DQ2/ Da8 positivity.

Aim Review the possible impact of ESPGHAN guidelines on the number of patients requiring histological assessment for CD.

Methods 3 year retrospective review of serology and histology of children screened for CD.

Results January 2009 - January 2012, 729 children screened. 32 positve with normal IgA levels.

Conclusion All but 1 patient with high anti-tTG levels $(>10 \mathrm{X}$ ) had characteristic histological changes. Anti-tTG levels $<10 \mathrm{X}$ normal range in all samples from January $2010-2012$ and $68 \%$ of all positive samples. Our results suggest that in most cases histological assessment will continue to play an important role in the diagnosis of CD. A multicentre prospective study on $\mathrm{CD}$ is currently underway.

\section{Abstract G206(P) Table 1}

\begin{tabular}{llll}
\hline \multicolumn{5}{c}{ Group 1 (January 2009-2010) anti-tTG $<12 \mathrm{U} / \mathrm{ml}$} \\
\hline anti-tTG (U/ml) & $\mathbf{N}=\mathbf{1 9}$ & Histology positive & Histology negative \\
\hline $12-18$ & 2 & 1 & 1 \\
$18-60$ & 4 & 2 & 2 \\
$60-100$ & 3 & 3 & 0 \\
$>120$ & 10 & 8 & 1 \\
\hline \multicolumn{5}{c}{ Group 2 (January 2010-2012) anti-tTG $<10 \mathrm{U} / \mathrm{mL}$} \\
\hline N-10 & $\mathbf{N}=\mathbf{1 3}$ & \\
$10-50$ & 3 & 3 & 0 \\
$50-100$ & 6 & 4 & 0 \\
\hline
\end{tabular}

\section{REFERENCE}

1. Husby et al. European Society for Pediatric Gastroenterology, Hepatology, and Nutrition Guidelines for the Diagnosis of Coeliac Disease. J Pediatr Gastroenterol Nutr 2012; 54 (1):136-160

\section{Young Persons Special Interest Group/Child Public Health Interest Group}

\section{G207 IS THERE A LINK BETWEEN ADHD AND SOCIAL DEPRIVATION?}

doi:10.1136/archdischild-2013-304107.219

L Apperley, R Mittal. Department of Community Paediatrics, Countess of Chester Hospital NHS Foundation Trust, Chester, UK

Aim Attention Deficit Hyperactivity Disorder (ADHD) is a neurodevelopmental disorder that affects approximately 4-6\% of schoolaged children. Research into the aetiology of ADHD has focussed on genetic and biological factors, with much less information on environment and social aspects. There is a general perception that 